\title{
Modeling of Tilting-Pad Journal Bearings with Transfer Functions
}

\author{
J.A. VÁZQUEZ* and L.E. BARRETT ${ }^{\dagger}$ \\ Department of Mechanical and Aerospace Engineering, School of Engineering and Applied Science, \\ University of Virginia, Charlottesville, VA 22903, USA
}

(Received in final form 6 July 1999)

\begin{abstract}
Tilting-pad journal bearings are widely used to promote stability in modern rotating machinery. However, the dynamics associated with pad motion alters this stabilizing capacity depending on the operating speed of the machine and the bearing geometric parameters, particularly the bearing preload. In modeling the dynamics of the entire rotor-bearing system, the rotor is augmented with a model of the bearings. This model may explicitly include the pad degrees of freedom or may implicitly include them by using dynamic matrix reduction methods. The dynamic reduction models may be represented as a set of polynomials in the eigenvalues of the system used to determine stability. All tilting-pad bearings can then be represented by a fixed size matrix with polynomial elements interacting with the rotor. This paper presents a procedure to calculate the coefficients of polynomials for implicit bearing models. The order of the polynomials changes to reflect the number of pads in the bearings. This results in a very compact and computationally efficient method for fully including the dynamics of tilting-pad bearings or other multiple degrees of freedom components that interact with rotors. The fixed size of the dynamic reduction matrices permits the method to be easily incorporated into rotor dynamic stability codes. A recursive algorithm is developed and presented for calculating the coefficients of the polynomials. The method is applied to stability calculations for a model of a typical industrial compressor.
\end{abstract}

Keywords: Rotating machine dynamics, Tilting-pad bearings, Dynamic reduction, Stability calculations, Transfer functions

\section{INTRODUCTION}

Barrett et al. (1988) and Brockett and Barrett (1993) showed the importance of including all dynamic coefficients of the tilting-pad bearing for stability analysis. In general a tilting-pad bearing, like that shown in Fig. 1, with $n$ pads has $2(5 n+4)$ coefficients. The variable number of coefficients could present a problem in some analysis programs since the number of equations of motion completely

\footnotetext{
* Corresponding author. E-mail: jose@virginia.edu.

† E-mail: leb@virginia.edu.
} 
representing the bearings will change with the number of pads. Brockett and Barrett (1993) showed that by using dynamic condensation equivalent stiffness and damping coefficients can be obtained which are functions of the complex frequency $s$. Thus the number of equations representing a tiltingpad bearing remains fixed although the bearing coefficients are variable throughout an analysis. The dynamic condensation method used by Brockett and Barrett (1993) follows that developed by Leung $(1978,1989)$ for structural analysis. Conversely, if a single input - single output transfer function is known as a ratio of polynomials with known coefficients, an equivalent mass-stiffnessdamping matrix model can be obtained (Maslen and Bielk, 1992).

The work presented here shows another approach. Instead of reducing the pad degrees of freedom for each complex frequency $s$, complex dynamic coefficients are calculated as a function of the complex variable $s$. The complex dynamic coefficients are represented by a ratio of polynomials or transfer functions. The advantage of this approach is that the resultant dynamic coefficient matrix is always $2 \times 2$. A different number of pads will only change the order of the polynomials but the general size of the matrix remains the same. This approach facilitates the inclusion of tilting-pad bearings in analysis tools because it requires few modifications from the standard eight coefficient hydrodynamic bearings. If the analysis tools already include magnetic bearing control transfer function models, this method can be used directly and no modifications are required (Brockett and Barrett, 1995). The transfer function representation of tilting-pad bearings also facilitates understanding the behavior of the bearing as a function of the vibration frequency.

\section{PROCEDURE}

Tilting-pad bearing models can be expressed by

$$
\begin{gathered}
{\left[\begin{array}{cc}
\underline{M} & 0 \\
0 & \underline{I}_{\mathrm{p}}
\end{array}\right]\left\{\begin{array}{l}
\underline{\ddot{u}} \\
\ddot{\delta}
\end{array}\right\}+\left[\begin{array}{ll}
\underline{C}_{u u} & \underline{C}_{u \delta} \\
\underline{C}_{\delta u} & \underline{C}_{\delta \delta}
\end{array}\right]\left\{\begin{array}{l}
\underline{\dot{u}} \\
\dot{\delta}
\end{array}\right\}} \\
+\left[\begin{array}{ll}
\underline{K}_{u u} & \underline{K}_{u \delta} \\
\underline{K}_{\delta u} & \underline{K}_{\delta \delta}
\end{array}\right]\left\{\begin{array}{l}
\underline{u} \\
\underline{\delta}
\end{array}\right\}=\left\{\begin{array}{l}
\underline{f} \\
\underline{0}
\end{array}\right\} .
\end{gathered}
$$

Dynamically reducing the pad degrees of freedom from Eq. (1) we get

$$
\begin{aligned}
s^{2}[M] & \{u\}+\left\{\left[s \underline{C}_{u u}+\underline{K}_{u u}\right]-\left[s \underline{C}_{u \delta}+\underline{K}_{u \delta}\right]\right. \\
\times & {\left[s^{2} \underline{I}_{\mathrm{p}}+s \underline{C}_{\delta \delta}+\underline{K}_{\delta \delta}\right]^{-1} } \\
\times & \left.\times\left[s \underline{C}_{\delta u}+\underline{K}_{\delta u}\right]\right\}\{u\}=\{f\},
\end{aligned}
$$
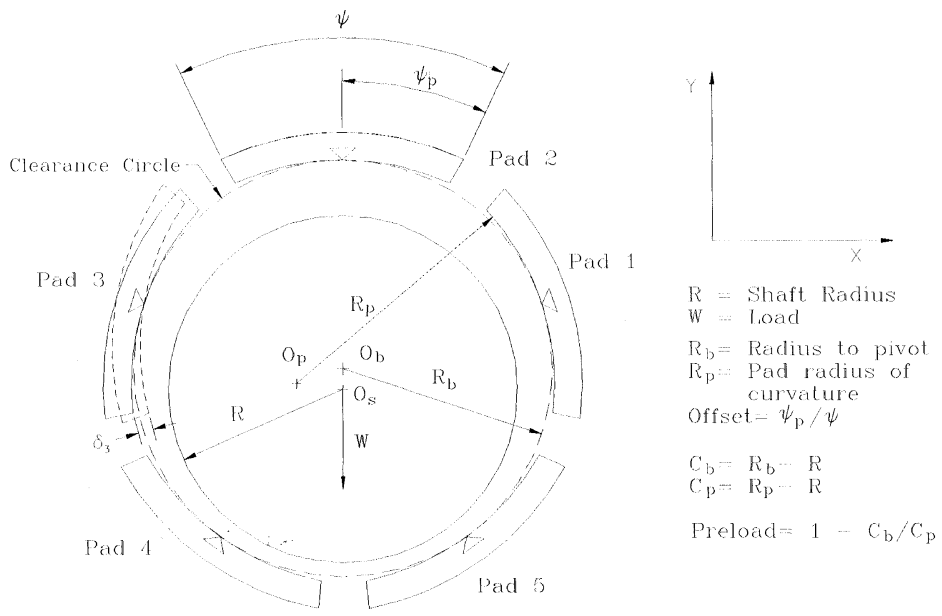

FIGURE 1 Schematic of a tilting-pad journal bearing. 
or

$$
s^{2}[M]\{u\}+\left[K_{\mathrm{eq}}(s)\right]\{u\}=\{f\}
$$

where

$$
\begin{aligned}
{\left[K_{\mathrm{eq}}(s)\right]=\{} & {\left[s C_{u u}+K_{u u}\right]-\left[s C_{u \delta}+K_{u \delta}\right] } \\
& \left.\times\left[s^{2} I_{\mathrm{p}}+s C_{\delta \delta}+K_{\delta \delta}\right]^{-1}\left[s C_{\delta u}+K_{\delta u}\right]\right\}
\end{aligned}
$$

Changing variables

$$
\begin{aligned}
A d_{2 i-1} & =a_{i}, \\
A d_{2 i} & =a_{i}, \\
B d_{2 i-1} & =b_{i}, \quad i=1 \ldots n . \\
B d_{2 i} & =d_{i}, \\
D d_{2 i-1} & =e_{i}, \\
D d_{2 i} & =f_{i},
\end{aligned}
$$

For an $n$ pad bearing

$\left[K_{\mathrm{eq}}(s)\right]=\left[\begin{array}{ll}s C_{x x}+K_{x x} & s C_{x y}+K_{x y} \\ s C_{y x}+K_{y x} & s C_{y y}+K_{y y}\end{array}\right]-\left[\begin{array}{l}\sum_{i=1}^{n} \frac{\left(s C_{x \delta_{i}}+K_{x \delta_{i}}\right)\left(s C_{\delta_{i} x}+K_{\delta_{i} x}\right)}{s^{2} I_{\mathrm{p}_{i}}+s C_{\delta_{i} \delta_{i}}+K_{\delta_{i} \delta_{i}}} \sum_{i=1}^{n} \frac{\left(s C_{x \delta_{i}}+K_{x \delta_{i}}\right)\left(s C_{\delta_{i} y}+K_{\delta_{i} y}\right)}{s^{2} I_{\mathrm{p}_{i}}+s C_{\delta_{i} \delta_{i}}+K_{\delta_{i} \delta_{i}}} \\ \sum_{i=1}^{n} \frac{\left(s C_{y \delta_{i}}+K_{y \delta_{i}}\right)\left(s C_{\delta_{i} x}+K_{\delta_{i} x}\right)}{s^{2} I_{\mathrm{p}_{i}}+s C_{\delta_{i} \delta_{i}}+K_{\delta_{i} \delta_{i}}} \sum_{i=1}^{n} \frac{\left(s C_{y \delta_{i}}+K_{y \delta_{i}}\right)\left(s C_{\delta_{i} y}+K_{\delta_{i} y}\right)}{s^{2} I_{\mathrm{p}_{i}}+s C_{\delta_{i} \delta_{i}}+K_{\delta_{i} \delta_{i}}}\end{array}\right]$

The $l, m$ equivalent complex bearing coefficients can be written as

$$
\begin{aligned}
K_{\mathrm{eq}_{l, m}}(s)= & S C_{l m}+K_{l m} \\
& -\sum_{i=1}^{n} \frac{\left(s C_{l \delta_{i}}+K_{l \delta_{i}}\right)\left(s C_{\delta_{i} m}+K_{\delta_{i} m}\right)}{s^{2} I_{\mathrm{p}_{i}}+s C_{\delta_{i} \delta_{i}}+K_{\delta_{i} \delta_{i}}} .
\end{aligned}
$$

Using partial-fraction expansion the summation term can be written as

$$
\begin{gathered}
\sum_{i=1}^{n} \frac{\left(s C_{l \delta_{i}}+K_{l \delta_{i}}\right)\left(s C_{\delta_{i} m}+K_{\delta_{i} m}\right)}{s^{2} I_{\mathrm{p}_{i}}+s C_{\delta_{i} \delta_{i}}+K_{\delta_{i} \delta_{i}}} \\
=\sum_{i=1}^{n}\left(\frac{s a_{i}+b_{i}}{s+e_{i}}+\frac{s a_{i}+d_{i}}{s+f_{i}}\right)
\end{gathered}
$$

where

$$
\begin{aligned}
A_{i} & =\frac{C_{l \delta_{i}} C_{\delta_{i} m}}{I_{\mathrm{p}_{i}}}, \quad e_{i}=D_{i}+\sqrt{D_{i}^{2}-E_{i}}, \\
B_{i} & =\frac{C_{l \delta_{i}} K_{\delta_{i} m}+K_{l \delta_{i}} C_{\delta_{i} m}}{I_{\mathrm{p}_{i}}}, \quad f_{i}=D_{i}-\sqrt{D_{i}^{2}-E_{i}}, \\
C_{i} & =\frac{K_{l \delta_{i}} K_{\delta_{i} m}}{I_{\mathrm{p}_{i}}}, \quad d_{i}=\frac{f_{i}\left[B_{i}-a_{i}\left(e_{i}+f_{i}\right)\right]-C_{i}}{\left(f_{i}-e_{i}\right)}, \\
D_{i} & =\frac{C_{\delta_{i} \delta_{i}}}{2 I_{\mathrm{p}_{i}}}, \quad b_{i}=\frac{C_{i}-d_{i} e_{i}}{f_{i}}, \\
E_{i} & =\frac{K_{\delta_{i} \delta_{i}}}{I_{\mathrm{p}_{i}}}, \quad a_{i}=c_{i}=\frac{A_{i}}{2} .
\end{aligned}
$$

Then Eq. (7) becomes

$$
\begin{aligned}
& \sum_{i=1}^{n} \frac{\left(s C_{l \delta_{i}}+K_{l \delta_{i}}\right)\left(s C_{\delta_{i} m}+K_{\delta_{i} m}\right)}{s^{2} I_{\mathrm{p}_{i}}+s C_{\delta_{i} \delta_{i}}+K_{\delta_{i} \delta_{i}}} \\
& \quad=\sum_{i=1}^{2 n} \frac{s A d_{i}+B d_{i}}{s+D d_{i}}
\end{aligned}
$$

or

$$
\begin{aligned}
& \sum_{i=1}^{n} \frac{\left(s C_{l \delta_{i}}+K_{l \delta_{i}}\right)\left(s C_{\delta_{i} m}+K_{\delta_{i} m}\right)}{s^{2} I_{\mathrm{p}_{i}}+s C_{\delta_{i} \delta_{i}}+K_{\delta_{i} \delta_{i}}} \\
& \quad=\frac{f_{2 n} s^{2 n}+f_{2 n-1} s^{2 n-1}+\cdots+f_{1} s+f_{0}}{g_{2 n} s^{2 n}+g_{2 n-1} s^{2 n-1}+\cdots+g_{1} s+g_{0}} .
\end{aligned}
$$

After some manipulation it can be shown that

$$
\begin{aligned}
& g_{2 n}=1, \\
& g_{2 n-1}=\sum_{i=1}^{2 n} D d_{i}, \\
& g_{2 n-2}=\sum_{i=1}^{2 n-1} D d_{i} \sum_{j=1+1}^{2 n} D d_{j}, \\
& g_{2 n-3}=\sum_{i=1}^{2 n-2} D d_{i} \sum_{j=i+1}^{2 n-1} D d_{j} \sum_{k=j+1}^{2 n} D d_{k},
\end{aligned}
$$




$$
\begin{aligned}
g_{2 n-4} & =\sum_{i=1}^{2 n-3} D d_{i} \sum_{j=i+1}^{2 n-2} D d_{j} \sum_{k=j+1}^{2 n-1} D d_{k} \sum_{l=k+1}^{2 n} D d_{l}, \\
\vdots & g_{i=1}^{2} D d_{i} \sum_{j=i+1}^{3} D d_{j} \sum_{k=j+1}^{4} D d_{k} \cdots \\
& \times \sum_{r_{2 n-1}=r_{2 n-2}+1}^{2 n} D d_{r_{2 n-1}}, \\
g_{0}= & \sum_{i=1}^{1} D d_{i} \sum_{j=i+1}^{2} D d_{j} \sum_{k=j+1}^{3} D d_{k} \cdots \sum_{r_{2 n}=r_{2 n-1}+1}^{2 n} D d_{r_{2 n}} \\
= & \prod_{i=1}^{2 n} D d_{i},
\end{aligned}
$$

the numerator in Eq. (11) follows a similar pattern as

$$
\begin{aligned}
& f_{2 n}=\sum_{i=1}^{2 n} A d_{i}, \\
& f_{2 n-1}=\sum_{i=1}^{2 n}\left(B d_{i}+A d_{i} \sum_{\substack{j=1 \\
j \neq i}}^{2 n} D d_{j}\right), \\
& f_{2 n-2}=\sum_{i=1}^{2 n}\left(B d_{i} \sum_{\substack{j=1 \\
j \neq i}}^{2 n} D d_{j}+A d_{i} \sum_{\substack{j=1 \\
j \neq i}}^{2 n-1} D d_{j} \sum_{\substack{k=j+1 \\
k \neq i}}^{2 n} D d_{k}\right) \text {, } \\
& f_{2 n-3}=\sum_{i=1}^{2 n}\left(B d_{i} \sum_{\substack{j=1 \\
j \neq i}}^{2 n-1} D d_{j} \sum_{\substack{k=j+1 \\
k \neq i}}^{2 n} D d_{k}\right. \\
& \left.+A d_{i} \sum_{\substack{j=1 \\
j \neq i}}^{2 n-2} D d_{j} \sum_{\substack{k=j+1 \\
k \neq i}}^{2 n-1} D d_{k} \sum_{\substack{l=k+1 \\
l \neq i}}^{2 n} D d_{l}\right) \\
& f_{1}=\sum_{i=1}^{2 n}\left(B d_{i} \sum_{\substack{j=1 \\
j \neq i}}^{2} D d_{j} \sum_{\substack{k=j+1 \\
k \neq i}}^{3} D d_{k} \cdots \sum_{\substack{r_{2 n-1}=r_{2 n-2}+1 \\
r_{2 n-1} \neq i}}^{2 n} D d_{r_{2 n-1}}\right. \\
& \left.+A d_{i} \prod_{\substack{j=1 \\
j \neq i}}^{2 n} D d_{j}\right)
\end{aligned}
$$

$$
f_{0}=\sum_{i=1}^{2 n} B d_{i} \prod_{\substack{j=1 \\ j \neq i}}^{2 n} D d_{j} .
$$

Therefore

$$
K_{\mathrm{eq}_{l, m}}(s)=\frac{f_{2 n+1}^{\prime} s^{2 n+1}+f_{2 n}^{\prime} s^{2 n}+\cdots+f_{1}^{\prime} s+f_{0}^{\prime}}{g_{2 n} s^{2 n}+g_{2 n-1} s^{2 n-1}+\cdots+g_{1} s+g_{0}},
$$

where

$$
\begin{aligned}
& f_{0}^{\prime}=K_{l m} g_{0}-f_{0} \\
& f_{1}^{\prime}=C_{l m} g_{0}+K_{l m} g_{1}-f_{1}, \\
& \vdots \\
& f_{2 n-1}^{\prime}=C_{l m} g_{2 n-2}+K_{l m} g_{2 n-1}-f_{2 n-1}, \\
& f_{2 n}^{\prime}=C_{l m} g_{2 n-1}+K_{l m} g_{2 n}-f_{2 n}, \\
& f_{2 n+1}^{\prime}=C_{l m} g_{2 n}
\end{aligned}
$$

Numerically, the complex stiffness coefficients can be expressed as equivalent real stiffness and real damping coefficients for any oscillatory complex frequency, $s$, from the relationships:

$$
\begin{gathered}
k_{\mathrm{eq}_{l, m}}(s)=\operatorname{Re}\left(K_{\mathrm{eq}_{l, m}}(s)\right)-\frac{\operatorname{Re}(s)}{\operatorname{Im}(s)} \operatorname{Im}\left(K_{\mathrm{eq}_{l, m}}(s)\right), \\
c_{\mathrm{eq}_{l, m}}(s)=\frac{1}{\operatorname{Im}(s)} \operatorname{Im}\left(K_{\mathrm{eq}_{l, m}}(s)\right) .
\end{gathered}
$$

\section{NUMERICAL EXAMPLE}

The rotor used for this example, shown in Fig. 2, is an eight stage centrifugal compressor used for natural gas re-injection at an offshore drilling site, running at $5626 \mathrm{rpm}$. The rotor is approximately $2.8 \mathrm{~m}$ long and $954 \mathrm{~kg}$ with the mass center near the mid-span. Table I lists the model for the rotor. It is 


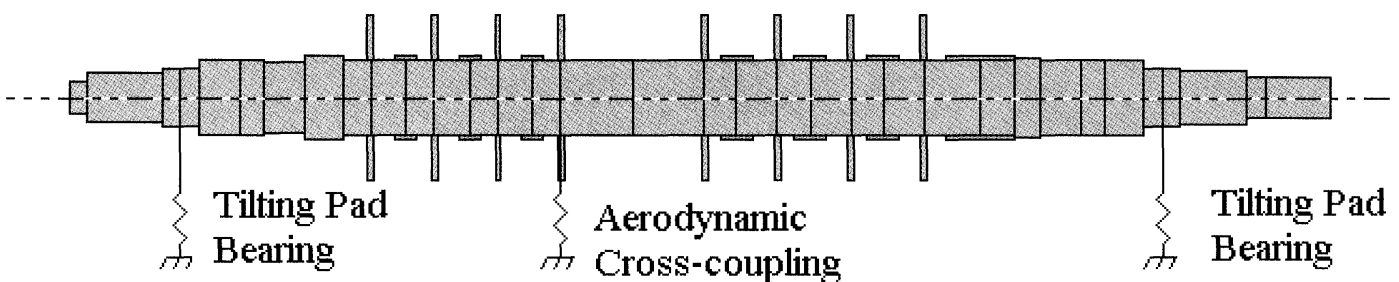

FIGURE 2 Rotor model.

TABLE I Rotor model

\begin{tabular}{|c|c|c|c|c|c|c|c|c|c|}
\hline $\begin{array}{l}\text { Station } \\
\text { no. }\end{array}$ & $\begin{array}{c}\text { Section } \\
\text { length }(\mathrm{m})\end{array}$ & $\begin{array}{c}\text { Outer } \\
\text { diameter }(\mathrm{m})\end{array}$ & $\begin{array}{c}\text { Transverse } \\
\text { moment of } \\
\text { inertia }\left(\mathrm{kg} \mathrm{m}^{2}\right)\end{array}$ & $\begin{array}{l}\text { Mass } \\
(\mathrm{kg})\end{array}$ & $\begin{array}{c}\text { Station } \\
\text { no. }\end{array}$ & $\begin{array}{c}\text { Section } \\
\text { length }(\mathrm{m})\end{array}$ & $\begin{array}{c}\text { Outer } \\
\text { diameter }(m)\end{array}$ & $\begin{array}{c}\text { Transverse } \\
\text { moment of } \\
\text { inertia }\left(\mathrm{kg} \mathrm{m}^{2}\right)\end{array}$ & Mass $(\mathrm{kg})$ \\
\hline 1 & 0.0345 & 0.0699 & 0.0002 & 72.60 & 19 & 0.0909 & 0.1702 & 0.0426 & 24.03 \\
\hline 2 & 0.1712 & 0.1107 & 0.0209 & 10.20 & 20 & 0.0721 & 0.1702 & 0.1251 & 48.84 \\
\hline 3 & 0.0381 & 0.1270 & 0.0229 & 12.92 & 21 & 0.0909 & 0.1702 & 0.0426 & 24.03 \\
\hline 4 & 0.0434 & 0.1270 & 0.0046 & 6.57 & 22 & 0.0721 & 0.1702 & 0.1251 & 48.84 \\
\hline 5 & 0.0881 & 0.1651 & 0.0199 & 13.92 & 23 & 0.0909 & 0.1702 & 0.0426 & 24.03 \\
\hline 6 & 0.0544 & 0.1681 & 0.0269 & 17.68 & 24 & 0.1273 & 0.1702 & 0.1464 & 56.00 \\
\hline 7 & 0.0897 & 0.1580 & 0.0249 & 17.28 & 25 & 0.0734 & 0.1702 & 0.0860 & 35.01 \\
\hline 8 & 0.0864 & 0.1824 & 0.0392 & 23.22 & 26 & 0.0587 & 0.1778 & 0.0277 & 17.87 \\
\hline 9 & 0.0625 & 0.1702 & 0.0357 & 21.08 & 27 & 0.0902 & 0.1681 & 0.0321 & 19.50 \\
\hline 10 & 0.0787 & 0.1702 & 0.1186 & 45.89 & 28 & 0.0544 & 0.1681 & 0.0287 & 18.27 \\
\hline 11 & 0.0625 & 0.1702 & 0.0334 & 20.36 & 29 & 0.0876 & 0.1651 & 0.0267 & 17.59 \\
\hline 12 & 0.0787 & 0.1702 & 0.1186 & 45.89 & 30 & 0.0434 & 0.1270 & 0.0197 & 13.52 \\
\hline 13 & 0.0625 & 0.1702 & 0.0334 & 20.36 & 31 & 0.0381 & 0.1270 & 0.0046 & 6.03 \\
\hline 14 & 0.0787 & 0.1702 & 0.1186 & 45.89 & 32 & 0.1445 & 0.1189 & 0.0186 & 11.74 \\
\hline 15 & 0.0625 & 0.1702 & 0.0334 & 20.36 & 33 & 0.0475 & 0.0950 & 0.0175 & 15.01 \\
\hline 16 & 0.1600 & 0.1702 & 0.1585 & 56.50 & 34 & 0.1400 & 0.0935 & 0.0092 & 14.51 \\
\hline 17 & 0.1600 & 0.1702 & 0.1124 & 41.72 & 35 & 0.0000 & 0.0935 & 0.0082 & 8.48 \\
\hline 18 & 0.0721 & 0.1702 & 0.1610 & 57.59 & & & & & \\
\hline
\end{tabular}

composed of 35 mass stations with identical tiltingpad bearings acting at nodes 4 and 31 . The complete set of tilting-pad bearing coefficients is shown in Table II. The stiffness and damping coefficients for these bearings can be calculated by using several methods (Shapiro and Colsher, 1977; Branagan, 1988). The geometry of the bearings in this analysis are given in Table III. Aerodynamic cross-coupling was assumed to act at station 16 with a value of $2.539 \times 10^{6} \mathrm{~N} / \mathrm{m}$. A stability analysis is performed for this rotor using synchronously reduced coefficients for the bearings assuming that $s=i \omega_{\mathrm{s}}$ in Eq. (5), where $\omega_{\mathrm{s}}$ is the shaft spin frequency; a method often employed in rotor stability calculations. The analysis is repeated using the transfer function representation of the bearings calculated using the algorithm presented in this paper. The synchronously reduced bearing coefficients are shown in Table IV. Table $\mathrm{V}$ shows the coefficients of the four transfer functions obtained from the coefficients of Table II using the method described in this paper (Eq. (12) and (15)).

\section{DISCUSSION OF RESULTS}

The eigenvalues obtained using the frequency dependent transfer function representation proposed in this paper are shown in Table VI. The 
TABLE II Tilting-pad bearing coefficients

\begin{tabular}{|c|c|c|c|c|c|}
\hline \multicolumn{6}{|c|}{ Translational coefficients } \\
\hline$K_{x x}(\mathrm{~N} / \mathrm{m})$ & $6.08 \mathrm{E}+07$ & & $C_{x x}(\mathrm{Ns} / \mathrm{m})$ & $2.65 \mathrm{E}+05$ & \\
\hline$K_{x y}(\mathrm{~N} / \mathrm{m})$ & $7.33 \mathrm{E}+07$ & & $C_{x y}(\mathrm{Ns} / \mathrm{m})$ & $-3.10 \mathrm{E}+04$ & \\
\hline$K_{y x}(\mathrm{~N} / \mathrm{m})$ & $-1.83 E+08$ & & $C_{y x}(\mathrm{~N} \mathrm{~s} / \mathrm{m})$ & $-3.07 \mathrm{E}+04$ & \\
\hline$K_{y y}(\mathrm{~N} / \mathrm{m})$ & $2.19 \mathrm{E}+08$ & & $C_{y y}(\mathrm{~N} \mathrm{~s} / \mathrm{m})$ & $6.32 \mathrm{E}+05$ & \\
\hline \multicolumn{6}{|c|}{ Pad coefficients } \\
\hline Pad number & 1 & 2 & 3 & 4 & 5 \\
\hline$K p_{x \delta}(\mathrm{N})$ & 1512 & -8610 & $-2.35 \mathrm{E}+06$ & $1.28 \mathrm{E}+06$ & $2.44 \mathrm{E}+06$ \\
\hline$K p_{\delta x}(\mathrm{~N})$ & -4168 & -2929 & $1.03 \mathrm{E}+05$ & $1.28 \mathrm{E}+06$ & $-1.95 \mathrm{E}+04$ \\
\hline$K p_{y \delta}(\mathrm{N})$ & 9546 & 4387 & $-9.06 \mathrm{E}+05$ & $-1.10 \mathrm{E}+07$ & $-6.49 \mathrm{E}+05$ \\
\hline$K p_{\delta y}(\mathrm{~N})$ & 1727 & -3432 & $-1.09 \mathrm{E}+05$ & $8.45 \mathrm{E}+04$ & $1.49 \mathrm{E}+05$ \\
\hline$K p_{\delta \delta}(\mathrm{Nm})$ & 278 & 278 & 8595 & $8.16 \mathrm{E}+04$ & 8596 \\
\hline$C p_{x \delta}(\mathrm{N})$ & 1.201 & -6.839 & -132.338 & 1242.36 & 296.110 \\
\hline$C p_{\delta x}(\mathrm{~N})$ & 5.579 & -11.216 & -132.338 & 1242.36 & 296.110 \\
\hline$C p_{y \delta}(\mathrm{N})$ & 7.583 & 3.485 & -321.587 & -2315.94 & 182.384 \\
\hline$C p_{\delta y}(\mathrm{~N})$ & 13.608 & 9.509 & -321.587 & -2315.94 & 182.384 \\
\hline$C p_{\delta \delta}(\mathrm{Nm})$ & 0.221 & 0.221 & 16.825 & 78.890 & 16.825 \\
\hline
\end{tabular}

TABLE III Bearing characteristics

\begin{tabular}{ll}
\hline Number of pads & 5 \\
Bearing load & $4680 \mathrm{~N}$ \\
$m$ (preload) & 0.0 \\
Load direction & On pad \\
Radius & $63.5 \mathrm{~mm}$ \\
Radial clearance & $0.1016 \mathrm{~mm}$ \\
Length & $63.5 \mathrm{~mm}$ \\
Rotor speed & $5626 \mathrm{rpm}$ \\
Offset factor & 0.5 \\
Pad arc length & $60^{\circ}$ \\
Pad moment & $1.13 \times 10^{-4} \mathrm{~kg} / \mathrm{m}^{2}$ \\
\hline
\end{tabular}

difference in the eigenvalues obtained using full tilting-pad models and those obtained with synchronously reduced bearing coefficients can be determined by comparing Tables VI and VII. The real part of the eigenvalues in Table VI are more negative than the real part of the eigenvalues in Table VII. In this model, therefore, synchronously reduced bearing coefficients tends to over-predict stability. This is consistent with the findings by Barrett et al. (1988) and Brockett and Barrett (1993, 1995). In particular, synchronously reduced bearing coefficients predicted the system to be stable (all eigenvalues have negative real part) while the full tilting-pad models found the rotor to be unstable.
TABLE IV Synchronously reduced coefficients

\begin{tabular}{lc}
\hline$K_{x x}$ & $2.23 \times 10^{7} \mathrm{~N} / \mathrm{m}$ \\
$K_{x y}$ & $-1.50 \times 10^{5} \mathrm{~N} / \mathrm{m}$ \\
$K_{y x}$ & $1.01 \times 10^{5} \mathrm{~N} / \mathrm{m}$ \\
$K_{y y}$ & $1.61 \times 10^{8} \mathrm{~N} / \mathrm{m}$ \\
$C_{x x}$ & $1.69 \times 10^{5} \mathrm{~N} \mathrm{~s} / \mathrm{m}$ \\
$C_{x y}$ & $319.78 \mathrm{~N} \mathrm{~s} / \mathrm{m}$ \\
$C_{y x}$ & $-119.55 \mathrm{~N} \mathrm{~s} / \mathrm{m}$ \\
$C_{y y}$ & $3.56 \times 10^{5} \mathrm{~N} \mathrm{~s} / \mathrm{m}$ \\
\hline
\end{tabular}

When using the synchronously reduced bearing coefficients it is not possible to locate some of the eigenvalues in Table VI. The reason for this is that some of the dynamics of the system are neglected when using the synchronously reduced bearing coefficients. This changes the dynamic system being analyzed and different results are expected. In particular, eigenvalues 9-12 in Table VI are directly related to the natural frequencies of the bearing pads, while eigenvalues 3-6 in Table VI are the result of the dependency of the bearing coefficients on the eigenvalues. Figure 3 shows the effect of the reduction frequency on the equivalent coefficients. The coefficients are plotted as a ratio of the synchronously reduced coefficients. It is shown that the reduction frequency has a great effect on 
TABLE V Transfer functions representing the tilting-pad bearings

\begin{tabular}{ccccccccc}
\hline Power of $s$ & Num $_{x x}$ & $\operatorname{Den}_{x x}$ & Num $_{x y}$ & Den $_{x y}$ & Num $_{y x}$ & Den $_{y x}$ & Num $_{y y}$ & Den $_{y y}$ \\
\hline 11 & $2.650 \mathrm{E}+05$ & & $-3.096 \mathrm{E}+04$ & & $-3.072 \mathrm{E}+04$ & & $6.324 \mathrm{E}+05$ & \\
10 & $2.504 \mathrm{E}+11$ & $1.000 \mathrm{E}+00$ & $-6.268 \mathrm{E}+09$ & $1.000 \mathrm{E}+00$ & $-6.291 \mathrm{E}+09$ & $1.000 \mathrm{E}+00$ & $5.839 \mathrm{E}+11$ & $1.000 \mathrm{E}+00$ \\
9 & $5.734 \mathrm{E}+16$ & $1.000 \mathrm{E}+06$ & $-2.264 \mathrm{E}+14$ & $1.000 \mathrm{E}+06$ & $-2.940 \mathrm{E}+14$ & $1.000 \mathrm{E}+06$ & $1.332 \mathrm{E}+17$ & $1.000 \mathrm{E}+06$ \\
8 & $4.010 \mathrm{E}+21$ & $2.349 \mathrm{E}+11$ & $3.441 \mathrm{E}+18$ & $2.349 \mathrm{E}+11$ & $-5.704 \mathrm{E}+18$ & $2.349 \mathrm{E}+11$ & $9.322 \mathrm{E}+21$ & $2.349 \mathrm{E}+11$ \\
7 & $2.271 \mathrm{E}+25$ & $1.674 \mathrm{E}+16$ & $2.811 \mathrm{E}+22$ & $1.674 \mathrm{E}+16$ & $-2.867 \mathrm{E}+22$ & $1.674 \mathrm{E}+16$ & $5.282 \mathrm{E}+25$ & $1.674 \mathrm{E}+16$ \\
6 & $6.774 \mathrm{E}+28$ & $9.598 \mathrm{E}+19$ & $9.560 \mathrm{E}+25$ & $9.598 \mathrm{E}+19$ & $-7.730 \mathrm{E}+25$ & $9.598 \mathrm{E}+19$ & $1.600 \mathrm{E}+29$ & $9.598 \mathrm{E}+19$ \\
5 & $1.233 \mathrm{E}+32$ & $2.866 \mathrm{E}+23$ & $1.776 \mathrm{E}+29$ & $2.866 \mathrm{E}+23$ & $-1.221 \mathrm{E}+29$ & $2.866 \mathrm{E}+23$ & $2.984 \mathrm{E}+32$ & $2.866 \mathrm{E}+23$ \\
4 & $1.460 \mathrm{E}+35$ & $5.185 \mathrm{E}+26$ & $1.949 \mathrm{E}+32$ & $5.185 \mathrm{E}+26$ & $-1.167 \mathrm{E}+32$ & $5.185 \mathrm{E}+26$ & $3.690 \mathrm{E}+35$ & $5.185 \mathrm{E}+26$ \\
3 & $1.106 \mathrm{E}+38$ & $6.001 \mathrm{E}+29$ & $1.133 \mathrm{E}+35$ & $6.001 \mathrm{E}+29$ & $-5.846 \mathrm{E}+34$ & $6.001 \mathrm{E}+29$ & $2.981 \mathrm{E}+38$ & $6.001 \mathrm{E}+29$ \\
2 & $5.066 \mathrm{E}+40$ & $4.280 \mathrm{E}+32$ & $2.504 \mathrm{E}+37$ & $4.280 \mathrm{E}+32$ & $-1.141 \mathrm{E}+37$ & $4.280 \mathrm{E}+32$ & $1.524 \mathrm{E}+41$ & $4.280 \mathrm{E}+32$ \\
1 & $1.377 \mathrm{E}+43$ & $1.645 \mathrm{E}+35$ & $-5.776 \mathrm{E}+37$ & $1.645 \mathrm{E}+35$ & $-5.776 \mathrm{E}+37$ & $1.645 \mathrm{E}+35$ & $4.508 \mathrm{E}+43$ & $1.645 \mathrm{E}+35$ \\
0 & $1.888 \mathrm{E}+45$ & $2.542 \mathrm{E}+37$ & $3.956 \mathrm{E}+38$ & $2.542 \mathrm{E}+37$ & $3.956 \mathrm{E}+38$ & $2.542 \mathrm{E}+37$ & $5.853 \mathrm{E}+45$ & $2.542 \mathrm{E}+37$ \\
\hline
\end{tabular}

TABLE VI Eigenvalues using the transfer function representation of the tilting-pad bearings

\begin{tabular}{ccc}
\hline Eigenvalue no. & Damping exp. $\left(\mathrm{s}^{-1}\right)$ & Frequency $(\mathrm{rpm})$ \\
\hline 1 & -11.74 & 2008 \\
2 & 2.76 & 2101 \\
3 & -124.8 & 3234 \\
4 & -156.6 & 3707 \\
5 & -393.5 & 4889 \\
6 & -338.8 & 5005 \\
7 & -13.55 & 7994 \\
8 & -17.50 & 8135 \\
9 & -981.8 & 11690 \\
10 & -979.5 & 11700 \\
11 & -977.7 & 11710 \\
12 & -978.1 & 11710 \\
13 & -275.1 & 13550 \\
14 & -132.0 & 15420 \\
15 & -198.8 & 16430 \\
\hline
\end{tabular}

the value of the equivalent coefficients. Therefore the full dynamics of the bearing should be used for stability calculations.

\section{CONCLUSIONS}

It is possible to model a tilting-pad bearing with transfer functions using the algorithm presented here. The stability analysis shows that synchronously reduced coefficients can predict eigenvalues significantly different than those predicted using a
TABLE VII Eigenvalues using the synchronously reduced coefficients

\begin{tabular}{lcc}
\hline Eigenvalue no. & Damping exp. $\left(\mathrm{s}^{-1}\right)$ & Frequency $(\mathrm{rpm})$ \\
\hline 1 & -44.31 & 1996 \\
2 & -1.23 & 2122 \\
3 & -14.44 & 7977 \\
4 & -20.67 & 8147 \\
5 & -583.7 & 11430 \\
6 & -104.7 & 15640 \\
7 & -190.3 & 15850 \\
\hline
\end{tabular}

complete model of the tilting-pad bearings. It is shown that using the synchronously reduced bearing coefficients tends to over-predict the stability of the system while some eigenvalues are eliminated completely from the model. A case is presented where the synchronously reduced coefficients predicted the rotor to be stable while a complete model of the tilting-pad bearing predicted instability. The evidence shown in this work supports the requirement of using all dynamics of the bearing when performing stability analysis. The transfer function representation is a simple way to include all dynamics of the bearing in existing computer codes.

The algorithm presented in this paper is just one of many ways to calculate the coefficients of the transfer functions representing a tilting-pad bearing. This one was chosen partly because it is possible to write each coefficient independently. 

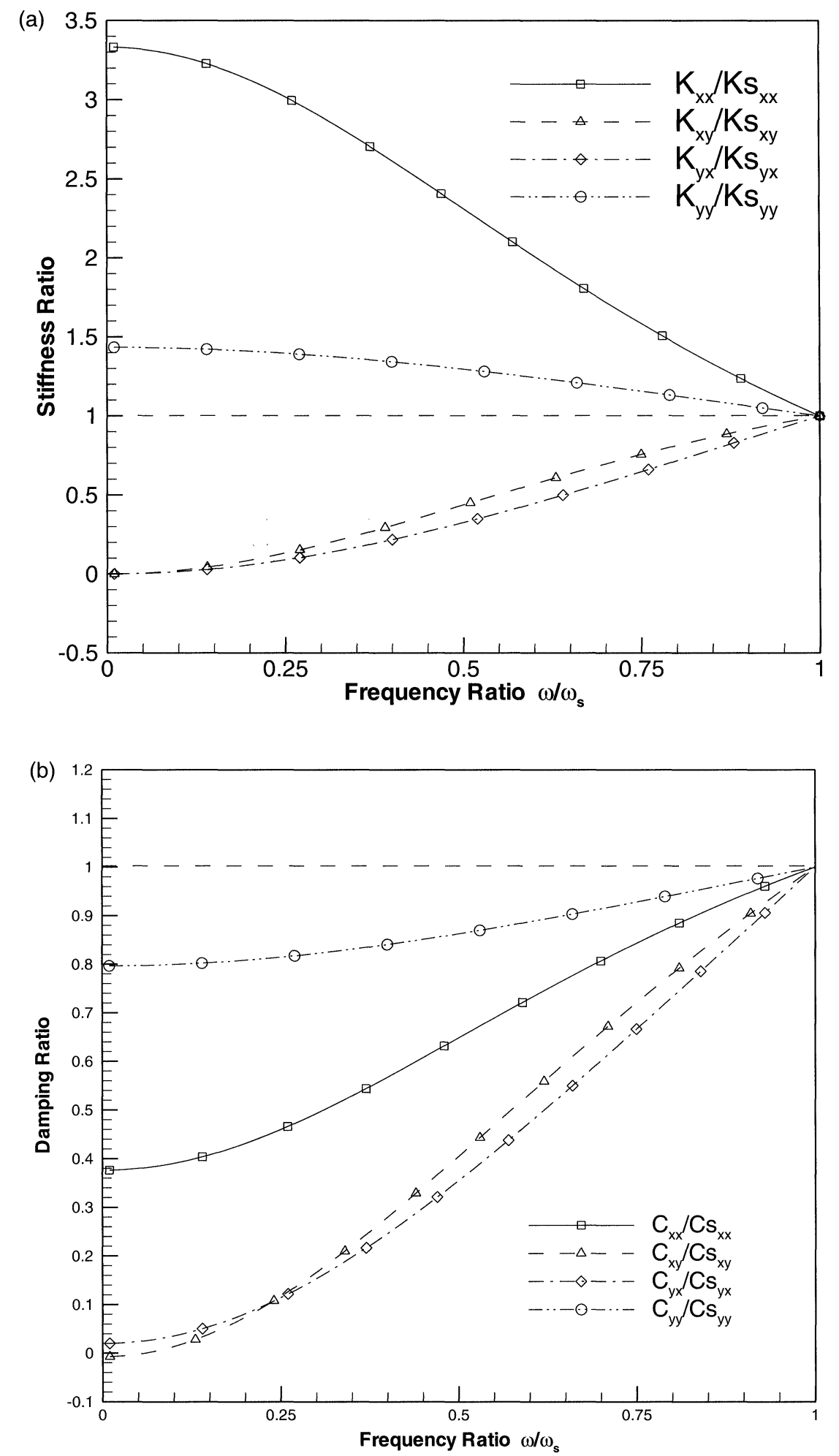

FIGURE 3 (a) Effect of the reduction frequency on the equivalent bearing stiffness coefficients. (b) Effect of the reduction frequency on the equivalent bearing damping coefficients. 


\section{NOMENCLATURE}

$$
\begin{gathered}
a_{i}, b_{i}, c_{i}, \\
d_{i}, e_{i}, f_{i} \\
A_{i}, B_{i}, C_{i}, \\
D_{i}, E_{i} \\
A d, B d, D d
\end{gathered}
$$$$
\left[c_{\text {eq }}(s)\right]
$$$$
C_{i j}
$$$$
C s_{i j}
$$$$
\left[C_{u u}\right]
$$$$
\left[C_{u \delta}\right],\left[C_{\delta u}\right]
$$$$
\left[C_{\delta \delta}\right]
$$$$
f_{i}^{\prime}, f_{i}, g_{i}
$$$$
\left[I_{\mathrm{p}}\right]
$$$$
\left[k_{\text {eq }}(s)\right]
$$$$
\left[K_{\mathrm{eq}}(s)\right]
$$$$
K_{\mathrm{eq}_{l, m}}(s)
$$$$
K_{i j}
$$

Coefficients for the partialfraction expansion Coefficients for the partialfraction expansion Change of variables to obtain the transfer function coefficients Equivalent bearing damping coefficient matrix as a function of the complex frequency $s, \mathrm{~N} \mathrm{~s} / \mathrm{m}$ The $i, j$ equivalent damping coefficients, reduced at a frequency different than synchronous (used in Fig. 3), $\mathrm{N} \mathrm{s} / \mathrm{m}$ The $i, j$ synchronously reduced damping coefficients (used in Fig. 3), $\mathrm{N} \mathrm{s} / \mathrm{m}$ Damping coefficient matrix for the shaft degrees of freedom, $\mathrm{Ns} / \mathrm{m}$ Cross-coupled damping coefficient matrices between the shaft and pad degrees of freedom, $\mathrm{N} \mathrm{s}$ Damping coefficient matrix for the pad degrees of freedom, N-m-s

Coefficients in the transfer function

Pad inertia matrix, $\mathrm{kg} \mathrm{m}^{2}$

Equivalent bearing stiffness coefficient matrix as a function of the complex frequency $s, \mathrm{~N} / \mathrm{m}$ Equivalent complex bearing coefficient matrix as a function of the complex frequency $s, \mathrm{~N} / \mathrm{m}$ The $l, m$ equivalent bearing coefficients, where $l$ or $m$ could be $x$ or $y$ The $i, j$ equivalent stiffness coefficients, reduced at a frequency different than synchronous (used in Fig. 3), N/m
$K s_{i j}$

$\left[K_{u u}\right]$

$\left[K_{u \delta}\right],\left[K_{\delta u}\right]$

$\left[K_{\delta \delta}\right]$

$m$

$[M]$

$n$

$s$

$\{u\}$

$x, y$

$\alpha$

$\delta_{1}, \delta_{2}, \ldots, \delta_{n}$

$\{\delta\}$

$\psi$

$\psi_{\mathrm{p}}$

$\omega$

$\omega_{s}$
The $i, j$ synchronously reduced stiffness coefficients (used in Fig. 3), N/m Stiffness coefficient matrix for the shaft degrees of freedom, $N / m$ Cross-coupled stiffness coefficient matrices between the shaft and pad degrees of freedom, $\mathrm{N}$ Stiffness coefficient matrix for the pad degrees of freedom, N-m Pad preload, dim. Mass matrix for the journal, $\mathrm{kg}$ Number of pads Complex frequency, $1 / \mathrm{s}$ Displacement vector for the shaft degrees of freedom $\equiv\left\{\begin{array}{ll}x y \\ y\end{array}\right\}^{\mathrm{T}}, \mathrm{m}$ Displacement coordinates of the shaft in the horizontal and vertical directions, $\mathrm{m}$ Pad offset factor, dim.

Rotation coordinates of pad $1,2, \ldots, n, \operatorname{dim}$.

Rotation vector for the pads degrees of freedom $\equiv$ $\left\{\delta_{1} \delta_{2} \ldots \delta_{n}\right\}^{\mathrm{T}}$, dim

Pad arc length, dim.

Pivot arc, dim.

Reduction frequency for the bearing coefficients (used in Fig. 3), rad/s

Spin speed of the rotor, $\mathrm{rad} / \mathrm{s}$

\section{References}

Barrett, L.E., Allaire, P.E. and Wilson, B.W., 1988, "The eigenvalue dependence of reduced tilting pad bearing stiffness and damping coefficients," Tribology Transactions, 31(4), 411-419.

Branagan, L.A., 1988, "Thermal analysis of fixed and tilting pad journal bearings including cross-film viscosity variations and deformations," Ph.D. Dissertation, University of Virginia.

Brockett, T.S. and Barrett, L.E., 1993, "Exact dynamic reduction of tilting-pad bearing models for stability analyses," STLE Tribology Transactions, 36(4), 581-588.

Brockett, T.S. and Barrett, L.E., 1995, "Magnetic bearing models with flexible supports in rotor stability analyses," 
Proceedings of the 1995 Design Engineering Technical Conferences, Vol. 3, Part B, pp. 1063-1072, Paper DE-Vol. 84-2. Leung, A.Y.-T., 1978, "An accurate method of dynamic condensation in structural analysis," International Journal for Numerical Methods in Engineering, 12, 1705-1715.

Leung, A.Y.-T., 1989, "Multilevel dynamic substructures," International Journal for Numerical Methods in Engineering, 28, $181-191$
Lund, J.W., 1964, "Spring and damping coefficients for the tilting-pad journal bearing," ASLE Transactions, 7, 342-352. Maslen, E.H. and Bielk, J.R., 1992, "A stability model for flexible rotors with magnetic bearings," Journal of Dynamic Systems, Measurement, and Control, 114, 172-175.

Shapiro, W. and Colsher, R., 1977, "Dynamic characteristics of fluid film bearings," Proceedings of the 6th Turbomachinery Symposium, Texas A\&M University, pp. 39-54 

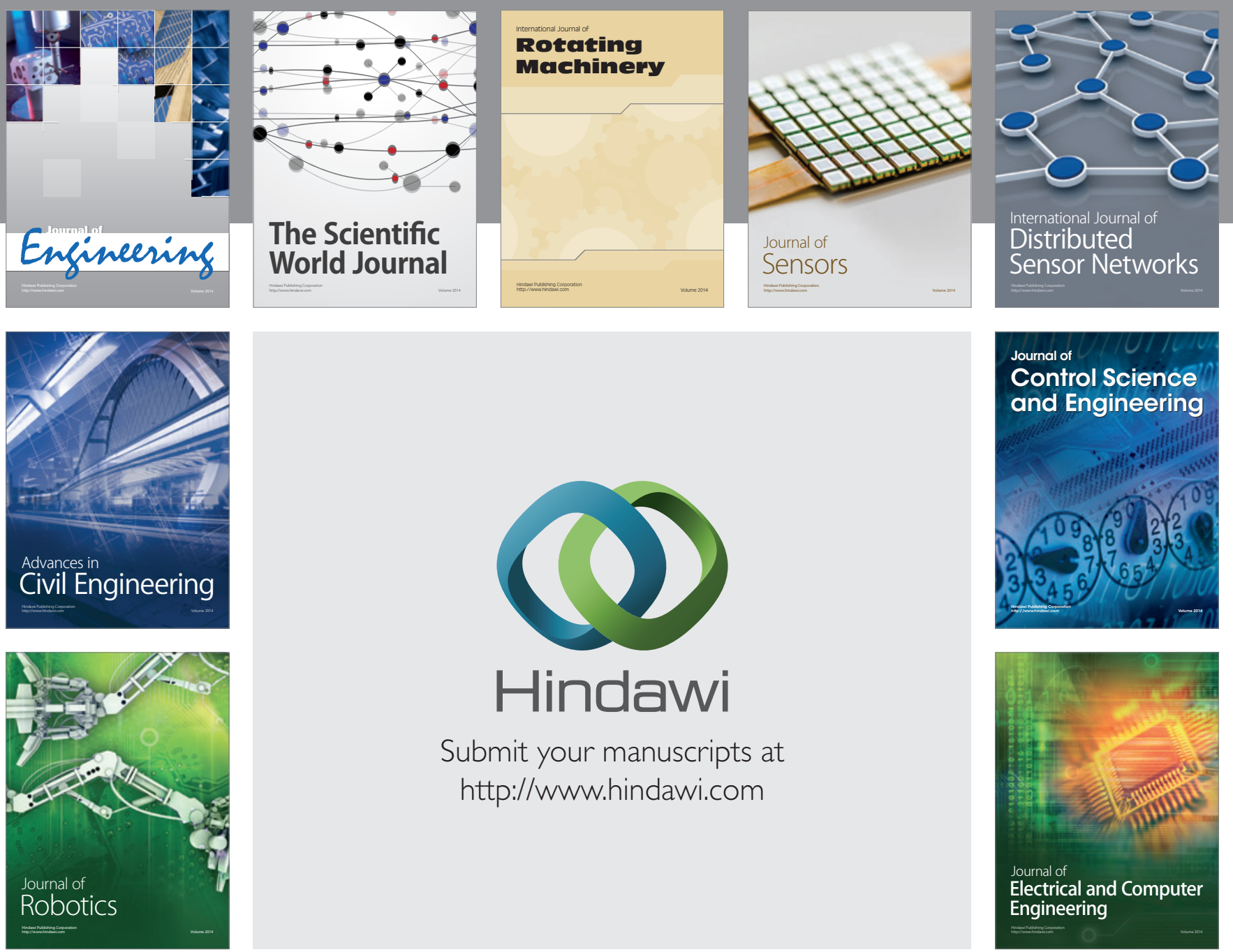

Submit your manuscripts at

http://www.hindawi.com
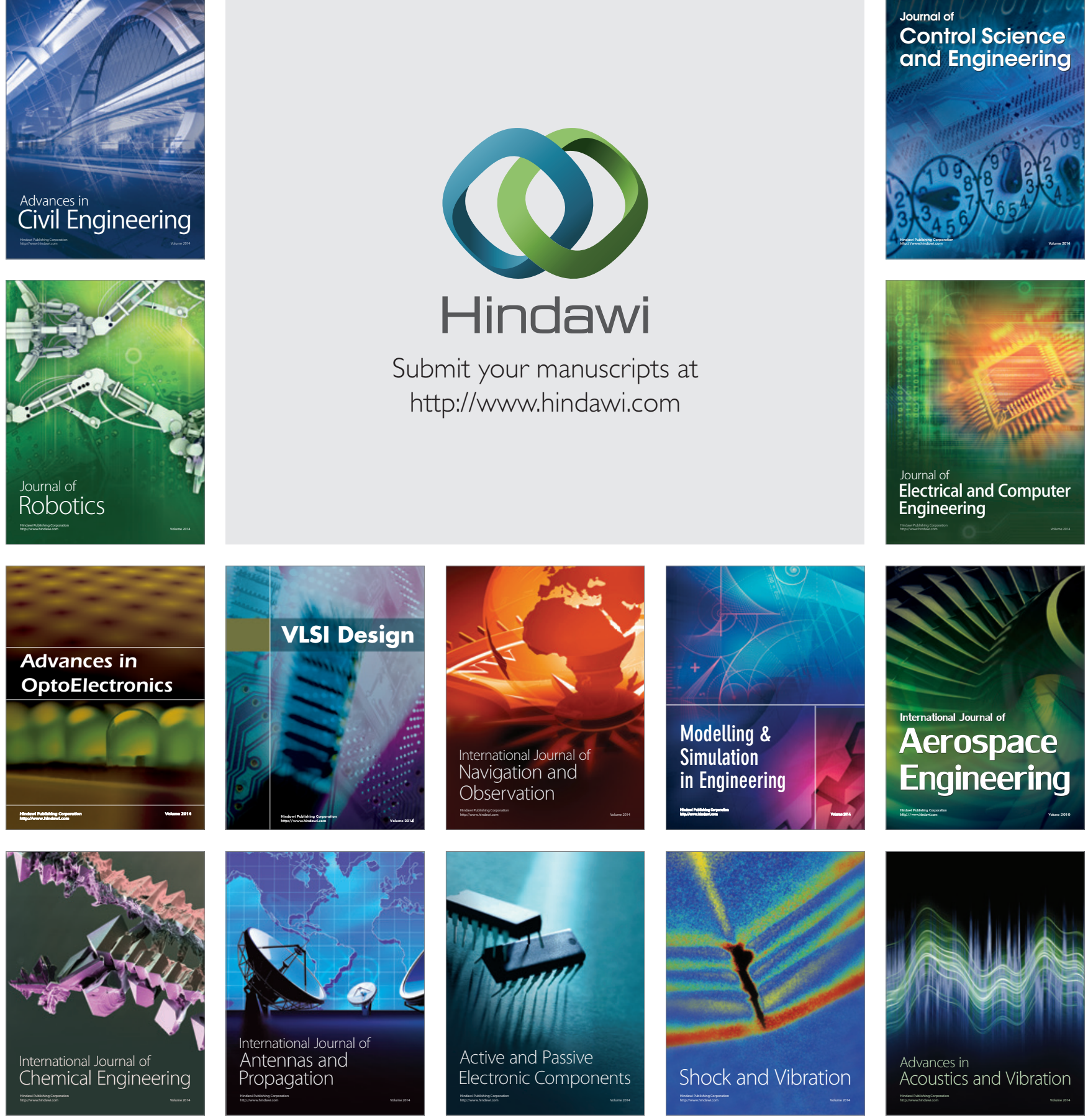